\title{
Territoire et interdépendance
}

\author{
Robert W. Cox
}

\section{(2) OpenEdition \\ Journals}

\section{Édition électronique}

URL : http://journals.openedition.org/conflits/249

DOI : $10.4000 /$ conflits.249

ISSN : $1777-5345$

Éditeur :

CCLS - Centre d'études sur les conflits lilberté et sécurité, L'Harmattan

\section{Édition imprimée}

Date de publication : 15 mai 1996

ISSN : 1157-996X

\section{Référence électronique}

Robert W. Cox, "Territoire et interdépendance », Cultures \& Conflits [En ligne], 21-22 | printemps-été 1996, mis en ligne le 15 mars 2006, consulté le 30 mars 2021. URL : http://journals.openedition.org/ conflits/249; DOI : https://doi.org/10.4000/conflits.249

Ce document a été généré automatiquement le 30 mars 2021.

Creative Commons License 


\title{
Territoire et interdépendance
}

\author{
Robert W. Cox
}

1 La question posée - l'existence et l'importance d'un international sans territoire intervient dans un contexte de scepticisme croissant à l'égard des théories conventionnelles en relations internationales. Le terme même de «relations internationales " semble fausser l'objet d'étude en le liant à une conception dépassée de l'ordre mondial, conception trop exclusivement Westphalienne.

2 Le défi économique à la territorialité Le premier défi a été lancé en économie politique. Il y eut sans doute plusieurs points de départ, mais un article publié en 1978 par Bernadette Madeuf et Charles-Albert Michalet $^{1}$ a particulièrement bien posé la distinction entre économie internationale et économie mondiale. L'économie internationale vise les flux de commerce, d'investissements, et de paiements à travers les frontières nationales, entre des économies nationales considérées comme des entités distinctes. L'économie mondiale est un système de production et de finance relativement récent qui échappe largement à la réglementation nationale. L'économie mondiale profite de la fragmentation territoriale du système des États en choisissant les lieux les plus propices pour diverses étapes du processus de production et les moins contraignants du point de vue fiscal. A la distinction entre économie internationale et économie mondiale s'ajoute une distinction entre économie réelle (production et commerce) et économie symbolique (la finance). On assiste à un découplement progressif entre les deux au point que la finance mondiale érode l'indépendance des États ; par son volume et ses aspects spéculatifs elle peut même déstabiliser l'économie réelle. Les conséquences se font sentir: dépérissement de la protection sociale, croissance du chômage, migrations internationales. Le territoire est donc devenu un appui moins propice pour la défense des conditions matérielles d'existence des moins favorisés. Mais, parce que ces moins favorisés n'ont pas accès à la puissance économique non-territoriale, le territoire est peut-être la seule base qui leur reste pour se défendre. Néanmoins, la croissance de l'économie mondiale (mondialisation économique néo-libérale) ne doit pas être conçue comme une tendance séculaire - dans le sens où plus il $\mathrm{y}$ a d'interdépendance, moins il $\mathrm{y}$ aura de territorialité - jusqu'à l'infini. Le rapport est plutôt dialectique. Tout d'abord, la défense du régime économique mondial repose en fin de compte sur la puissance territoriale. Si 
l'économique semble renforcer le principe non-territorial d'interdépendance, le politico-militaire repose toujours sur une base territoriale. La guerre du Golfe a démontré comment l'économie mondiale était vulnérable aux ambitions d'une puissance régionale (l'Irak) voulant utiliser une ressource indispensable à l'économie mondiale (le pétrole) à ses propres fins. Et ce fut la puissance territoriale politicomilitaire des États-Unis qui organisa la défense des intérêts de l'économie mondiale. Ensuite, la poussée de mondialisation économique peut provoquer, par ses effets négatifs sur la déstabilisation de l'économie réelle et les conditions sociales des plus vulnérables, un contre-mouvement à base territoriale. Jacques Delors a préconisé une régulation des marchés monétaires afin de créer une sphère de stabilité relative en Europe vis-à-vis de la turbulence monétaire mondiale. Ce serait une défense basée sur le territoire européen. Et les forces sociales voulant défendre les conditions matérielles de vie des couches subordonnées, ayant peu d'influence au niveau mondial, agissent de préférence sur l'état territorial ou éventuellement au niveau régional. Il y a donc une alternance entre les principes de territoire et d'interdépendance. Ces contradictions apparentes dans le domaine économique sont le reflet d'une contradiction plus large entre tendances mondiales d'homogénéisation et de différentiation. L'homogénéisation est poussée par une aspiration quasi-universelle vers la société de consommation. Par contre, nous sommes tous conscients - et les communications dans cet ouvrage le reflètent - d'une affirmation plus forte, plus agressive parfois, des identités diverses, qu'elles soient définies par l'ethnicité, par la religion, par la langue, par le sexe ou autre caractéristique donnée. En général, on peut poser comme hypothèse que l'homogénéisation vient d'en haut, des forces les plus puissantes, du capital et des communications de masse; et que l'affirmation des identités vient d'en bas, comme moyen de défense des moins puissants. Le résultat est un déséquilibre de forces: la mondialisation économique gagne, et en même temps accentue la polarisation sociale. Les moins puissants, les victimes de la polarisation sociale, s'entre-déchirent en conflits entre eux, s'affaiblissant en face des forces dominantes. L'international sans territoire des démunis ne pourrait se faire qu'en construisant un bloc contre-hégémonique surmontant leurs antagonismes tout en gardant leurs identités respectives. C'est le problème fondamental de la gauche au niveau national et international. Mais cet international contre-hégémonique serait fondé le plus probablement sur une agglomération de noyaux de résistance territoriaux. L'avenir du territoire La mise en question de l'État territorial nous amène à considérer d'autres schémas d'organisation politico-économique à venir. L'un, souvent évoqué, est la notion d'une organisation de type néo-médiévale avancée entre autres par Hedley Bull ${ }^{2}$. Elle modifie la notion de souveraineté en envisageant des autorités se chevauchant sur le même territoire, et divers territoires liés par des réseaux ayant chacun une certaine autonomie vis-à-vis des institutions territoriales (par analogie avec les ordres religieux). Cette notion est peut-être plus évoluée en Europe qu'ailleurs, les institutions macro-régionales coexistant avec une poussée de micro-régionalismes (Catalogne, Ecosse, Lombardie, etc.) sur un fond d'États historiques. Le contexte plus profond d'une refonte des institutions sur le plan régional et global comprend des forces et mouvements à très long terme, la restructuration de la longue durée dont parlait Fernand Braudel. Toutes ces forces et tous ces mouvements dépassent les frontières. Ils forment un système mondial d'avenir, dans lequel le rapport territoire/interdépendance évoluera. Parmi ces forces structurantes, $\mathrm{j}$ 'en mentionnerai cinq : La polarisation sociale. Cela se passe entre pays, entre unités territoriales, par exemple la dégradation de conditions en 
Afrique par rapport aux autres régions du monde. En cela, la polarisation conserve un aspect territorial. Mais elle se passe surtout à l'intérieur des sociétés nationales, moins aiguë dans certaines qui ont pu conserver quelque chose de la protection sociale, mais frappant quand même partout, dans les pays dits riches aussi bien que dans les pays pauvres $^{3}$. Les notions de centre et périphérie en économie mondiale qui avait autrefois une connotation géographique et donc territoriale, ont maintenant une signification de structuration sociale à travers les frontières géographiques. Le Tiers monde grandit à l'intérieur des pays les plus riches. Une décomposition très largement répandue de la société civile. Les peuples participent moins à la vie de leurs institutions, ils perdent confiance dans les classes politiques et leurs prétendus chefs, considérés comme corrompus et ne sachant pas comment résoudre les problèmes que confrontent les sociétés ${ }^{4}$. Dans certains cas extrêmes, dans le Tiers monde, les peuples tournent le dos aux Etats. Un économiste éthiopien, Fantu Cheru, parle d'une révolution silencieuse en Afrique où beaucoup de gens tâchent d'organiser leurs activités sur un plan local en dehors des circuits de l'État et des institutions internationales comme la Banque mondiale et le FMI considérés comme les ennemis du peuple ${ }^{5}$. Ils se replient sur des territoires minuscules où ils ont parfois de la peine à survivre face à des gangs armés. L'État n'existe presque plus. L'économie souterraine se répand aussi dans les pays plus riches comme mécanisme de défense contre la fiscalité de l'État et comme moyen de court-circuiter la bureaucratie. A la décomposition vient en réponse une tentative de recomposition de société civile et ceci d'une manière transterritoriale avec des mouvements écologiques et féministes, par exemple, mais aussi avec un renouveau de fascismes et de racismes ayant leurs propres réseaux transnationaux. Et puis il y a le monde clandestin peuplé par les services de renseignements, résidus de la guerre froide, le commerce des armes, la crise organisée, les cartels de la drogue, et les banques qui les servent. C'est un élément important mais difficile à peser dans la formation de nouvelles structures sociales et politiques. La constitution et la rivalité entre diverses formes d'organisation économique. Michel Albert a écrit un livre intéressant sur la rivalité entre capitalisme de type anglo-américain et ce qu'il appelle le type rhénan basé sur la notion de partenaires sociaux ${ }^{6}$. Il me semble que les grands débats sur l'avenir de l'Europe tournent en grande partie autour de cette rivalité. Nous pouvons voir aussi un type de capitalisme asiatique (décrit par Chalmers Johnson ${ }^{7}$ Fallows $\left.{ }^{8}\right)$. Au lieu d'adopter une conception de capitalisme monolithique triomphant sur le "socialisme réel», il serait plus réaliste de revenir à la notion d'économie substantive de Karl Polanyi ${ }^{9}$. Pour comprendre la diversité de l'organisation économique, surtout dans son incidence sur les rapports sociaux. Les grands espaces pourront être organisés très différemment notamment en ce qui concerne le contenu éthique et social de l'économie. Les impératifs de la biosphère. Récemment nous avons commencé à comprendre comment la biosphère était un acteur dans les affaires de l'humanité et non seulement un récipient passif de l'action humaine. Cela demande une refonte de la mentalité surtout en matière de pensée économique. En économie formelle, la nature, réduite à la terre, est soumise à la logique du marché. Mais la nature, par contre, possède sa propre logique, basée sur l'interdépendance de différentes formes de vie, humaine et non-humaine. Aussi longtemps que la logique économique ne résultait pas dans une déstabilisation de la nature, aussi longtemps qu'il y avait une marge de tolérance dans la nature pour supporter les conséquences de la logique économique, cette subordination de la nature au marché passait inaperçue. Maintenant les limites de cette tolérance sont à l'épreuve. La diminution de la couche 
d'ozone. Le réchauffement climatique, la perte de biodiversité, etc. posent des problèmes graves pour l'avenir de la biosphère. Une des implications est de repenser l'économie en la subordonnant à une science de la nature. Cela est plus qu'un problème intellectuel ; cela implique une révision de nos façons de produire et de notre modèle de consommation - nos manières de travailler et de vivre. La coexistence de civilisations. Dernièrement, dans un débat ouvert en relations internationales, les civilisations ont été représentées comme entités géopolitiques en conflit actuel ou potentiel ${ }^{10}$. Je crois que cette représentation est le reflet d'une mentalité formée par la guerre froide - la recherche d'un " autre » pour remplacer « l'autre » du communisme mondial. Une civilisation naît, bien sûr, dans un contexte géographique, elle est influencée par les conditions de son lieu d'origine. Mais son évolution l'éloigne de la géographie pour devenir un phénomène d'intersubjectivité. La coexistence de civilisations pose un problème de compréhension entre mentalités différentes face à des réalités communes. Deux civilisations existent souvent au sein du même territoire, et parfois à l'intérieur de la même personne. La difficulté de coexistence entre civilisations est moins un affrontement politico-militaire qu'une question de dominance et subordination morale et intellectuelle. La dominance procède par l'occultation de la pensée du subordonné. Deux mentalités coexistent, mais une seule est légitime et hégémonique. Le problème de coexistence entre civilisations est de faire ressortir ce qui était occulté. C'est avant tout un problème de reconnaissance. Et nous pouvons voir en cela le fondement éthique de l'international sans territoire. Une remarque finale sur l'ambiguïté de la notion de souveraineté à partir de la situation au Canada. On peut y observer trois variantes: Au Québec, on retrouve la notion d'Étatnation territorial de type européen. Il est intéressant de noter que l'aspect territorial date seulement de la "révolution tranquille" des années 1950. Avant cela, les francophones du Québec s'identifiaient comme "Canadiens ", les gens du pays ${ }^{11}$, tandis que les autres étaient « les Anglais », des étrangers plus récemment arrivés. Depuis, ces mêmes gens s'appellent "Québécois ", donc avec une identité territoriale, ce qui veut dire que les francophones au Canada en dehors du Québec sont plus ou moins oubliés ou mis à côté et que les anglophones et immigrants au Québec doivent être considérés des Québécois par principe de territorialité. Dans le reste du Canada, la notion de multiculturalisme a plus ou moins déplacé les anciens liens britanniques comme symbole d'identité. C'est une notion d'une collection de communautés, «Gandhienne » dirait Christophe Jaffrelot. Ce qui explique la résistance au modèle québécois Les anglophones voulant associer les Québécois dans le mélange multiculturel. La notion de souveraineté, vantée par les Québécois, paraît de plus en plus illusoire aux autres Canadiens quand on comprend que la politique gouvernementale est tributaire de la finance internationale et de ses liens dans la bourgeoisie financière canadienne. Enfin, il y a les peuples indigènes qui revendiquent une grande part de la surface territoriale du Canada, y compris au Québec. Ils montent une défense écologique contre l'exploitation de leurs terres ancestrales par le capitalisme moderne cherchant les minerais, le bois et le pouvoir hydroélectrique. "Souveraineté » pour les peuples indigènes signifie droit de gestion de leurs terres et autonomie de leur propre organisation communautaire à l'intérieur du Canada. Cela ne veut pas dire indépendance. En somme, les rapports Territoire-État-Peuple ou Nation-Souveraineté sont très flous et évolutifs. Ceci n'est qu'un exemple d'une situation beaucoup plus générale. Les exigences de l'économie mondiale limitent les pouvoirs effectifs des États. Il y a des forces puissantes à l'intérieur des pays qui accueillent favorablement ces 
influences transnationales pour former un consensus mondial néo-libéral dans ce que Gramsci aurait appelé une "révolution passive». Cela provoque des réactions de défense d'en bas. D'un côté, il y a l'économie noire ou informelle (discutée par François Constantin). De l'autre côté, il y a les mouvements sociaux qui maintenant agissent surtout en dehors des structures politiques conventionnelles. Et l'on en revient au thème de la décomposition et recomposition de la société civile et aux innovations et dangers dans ce processus. Il me semble que c'est là le problème clé dans la construction des autorités politiques d'avenir, dans «l'invention du monde » pour reprendre les mots de Denis Retaillé.

\section{NOTES}

1. Bernadette Madeuf, Charles-Albert Michalet, « A New Approach to International Economics », Revue Internationale des Sciences Sociales, vol. XXX, n 2, 1978.

2. Hedley Bull, The Anarchical Society. A Study of Order in World Politics, New York, Columbia University Press, 1977, pp. 264-276.

3. Voir par exemple John Kenneth Galbraith, The Culture of Contentment, Boston, Houghton Miflin, 1992.

4. L'idée est développée dans Jean-Marie Gehenno, La fin de la démocratie, Paris, Flammarion, 1993. Il constate le déclin de l'esprit civique, donc de la politique qui suppose un choix entre projets de société et le remplacement de la politique par la gestion des intérêts.

5. Cheru Fantu, The Silent Revolution in Africa. Debt. Development and Democracy, London, Zed Books, 1989.

6. Michel Albert, Capitalisme contre capitalisme, Paris, Seuil, 1991.

7. Chalmers Johnson, MITI and the Japanese Miracle, Stanford University Press, 1982.

8. James Fallows, Looking at the Sun. The Rise of the New East Asian Economic and Political System, New York, Pantheon, 1994.

9. Karl Polanyi, «The Economy as instituted process », in Polanyi et al. eds., Trade and Market in the Early Empires, Chicago, Henry Regnery, 1957.

10. Samuel Huntington, «The Clash of Civilizations? », Foreign Affairs 72 (3), Summer 1993, pp. 22-49.

11. «Gens du pays » est le titre d'une chanson de Gilles Vigneault qui est devenue l'hymne national officieux des Québecois.

INDEX

Mots-clés : territoire(s) et territorialité, Relations Internationales, pauvreté 